\title{
Retinal arterial macroaneurysms: a retrospective study of 40 patients
}

\author{
MICHAEL J LAVIN,' RONALD J MARSH,' STANLEY PEART,' \\ AND ALIM REHMAN
}

\begin{abstract}
From the 'Western Ophthalmic Hospital, Marylebone Road, London NW1; the ${ }^{2}$ Pickering Unit and Department of Medicine, St Mary's Hospital, London W1; and 'St Mary's Hospital Medical School, London WI
\end{abstract}

SUMmaRY We studied 40 patients with a total of 44 retinal arterial macroaneurysms. All patients were followed up for at least six months. Macroaneurysms (MAs) have variable clinical presentations and are still frequently misdiagnosed before fluorescein angiography. Haemorrhagic MAs were most frequently misdiagnosed $(75 \%)$, and had a sudden onset with a relatively poor visual outcome. Patients with these MAs had higher systolic blood pressures and significantly fewer associated retinal vein occlusions $(p<0.05)$ than other types of MA. Exudative MAs caused a gradual onset of symptoms, were frequently associated with retinal vein occlusions, and were the most frequent indication for laser treatment. Only one of 10 quiescent MAs subsequently developed significant exudation or haemorrhage. We confirm the association of MAs with retinal and systemic vascular disease. In addition we found that MA patients had a significantly higher blood packed cell volume (haematocrit) than controls $(p<0.05)$. Laser treatment significantly shortened the duration of MA patency $(\mathrm{p}=0 \cdot 006)$.

Retinal arterial macroaneurysms (MAs) are acquired aneurysmal dilatations of the retinal arteries which usually occur in elderly, hypertensive people. The term macroaneurysm was introduced in 1973 to distinguish aneurysms of the retinal arteries from those of capillaries and veins. ${ }^{1-3}$ Although Robertson's report was the first review, isolated cases of retinal arterial aneurysms have been described since the nineteenth century ${ }^{+7}$ and a number in the past decade. ${ }^{x-27}$. MAs appear as local arterial dilatations with variable degrees of artery wall hyalinisation and surrounding retinal exudate or haemorrhage. They are frequently associated with retinal vascular occlusions, ${ }^{1111} 1323$ retinal emboli, ${ }^{13}$ systemic hypertension, ${ }^{16}$ and vascular disease. ${ }^{16}$ Histological examination confirms the presence of a true aneurysm with vessel wall thickening, hyaline change, and elastotic degeneration. ${ }^{19} 26$

Most reports have not classified MAs, although Palestine and colleagues' study ${ }^{23}$ grouped MAs according to their position in relation to the vascular arcades and whether visual acuity was affected.

Correspondence to Mr Michacl Lavin. FRCS, Moorficlds Eyc Hospital, City Road, London EC1V 2PD
Nevertheless, visual loss is influenced not only by the proximity of the MA to the macula, but also by the presence of oedema, exudate, or haemorrhage and its severity, duration, and position. Since the MAs with visual loss and haemorrhage may have a different course and outcome than those with exudate, ${ }^{1121}$ a classification reflecting this may be useful.

Spalter $^{2+}$ has emphasised that MAs are often incorrectly diagnosed and regards them as a masquerade syndrome. The differential diagnosis includes Coats' disease, Leber's miliary aneurysms, von Hippel-Lindau syndrome, and very rarely venous macroaneurysms. It is important to differentiate MAs from retinal capillary aneurysms which may at times be large but do not arise from an artery and may therefore be treated without risk of vascular injury.

Photocoagulation of MAs was first described by Hudomel and Imre ${ }^{x}$ and has since been reported by several authors. ${ }^{162123-2527}$ Although visual acuity is often reported as improved after laser photocoagulation of the MAs with macular oedema or exudate, it may improve spontaneously. MAs are described as being obliterated within one to seven months of 
treatment, though no study has compared this with a large group of untreated cases.

Despite the fact that MAs are not rare, they are frequently misdiagnosed, the natural history is not clear, and only two reports have included more than 20 patients. Further information is required to define prognostic features and indications for and results of treatment. We were therefore prompted to review our series.

\section{Material and methods}

We retrospectively reviewed cases of angiographically proved retinal arterial macroaneurysms seen at the Western Ophthalmic Hospital, London, from 1973 to 1984. The cases were identified from a fluorescein punch card index. The patients' notes were studied, and where necessary patients were recalled for reexamination. Colour stereo photographs and fluorescein angiograms were examined and classified separately by two of the authors (MJL and RJM). Particular attention was paid to MA characteristics and positions, retinal vascular abnormalities, and to the presence of associated ocular disease. A thorough medical assessment was performed on 18 patients $(45 \%)$ in the medical clinic supervised by Sir Stanley Peart. Particular attention was paid to the cardiovascular examination, and most patients had electrocardiography, chest $x$-ray, full blood count, and tests of electrolytes and urea performed. The blood packed cell volume and systolic blood pressure at or within six weeks of presentation were available in a number of patients and were compared between groups and with age matched controls. All cases had a minimum of six months' follow-up.

Clinically and histologically MAs are characterised by a dynamic process of formation, enlargement with or without blood-retinal barrier disruption, and gradual spontaneous resolution after a variable time period. Since the extent of blood-retinal barrier disruption determines the nature of visual impairment, we classified MAs in terms of their barrier function:

Quiescent MAs, with haemorrhage or exudate extending for less than 1 disc diameter and sparing the macula (Fig. 1).

Exudative MAs, in which exudate is the major component measuring more than 1 disc diameter and is responsible for visual loss in cases combined with haemorrhage (Fig. 2).

Haemorrhagic MAs with haemorrhage extending more than 1 disc diameter, more extensive than any associated exudate, and responsible for visual loss (Fig. 3).

Argon laser photocoagulation was performed on 10 patients, five with exudative MAs and five with

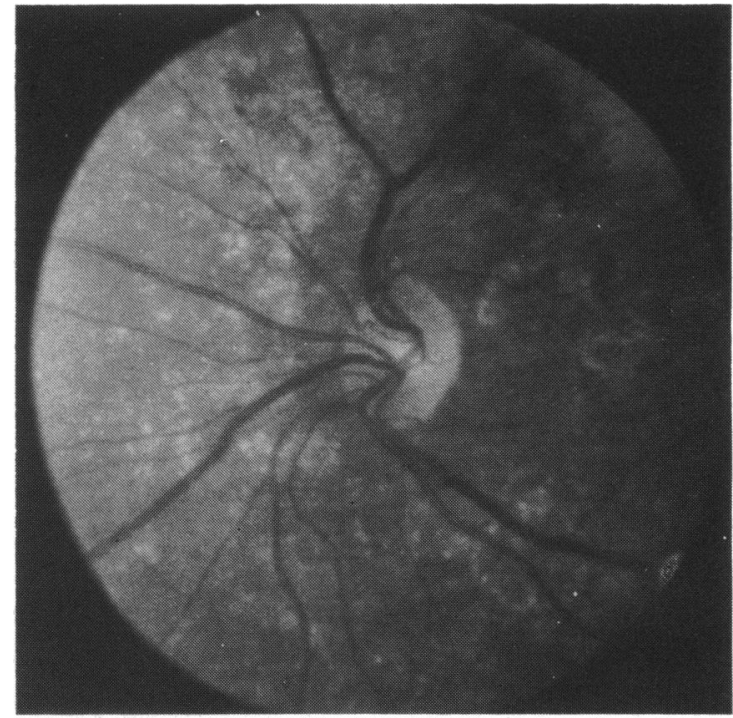

Fig. 1 Fundus photograph of a quiescent macroaneurysm, with minor local intraretinal haemorrhage.

haemorrhagic MAs. In the haemorrhagic group two MAs were treated because of the development of macular oedema or exudate, while three were treated to prevent further haemorrhage. The technique consisted of applying a ring of argon laser photocoagulation (Coherent 200 source) two or three burns in width to the retina immediately surrounding

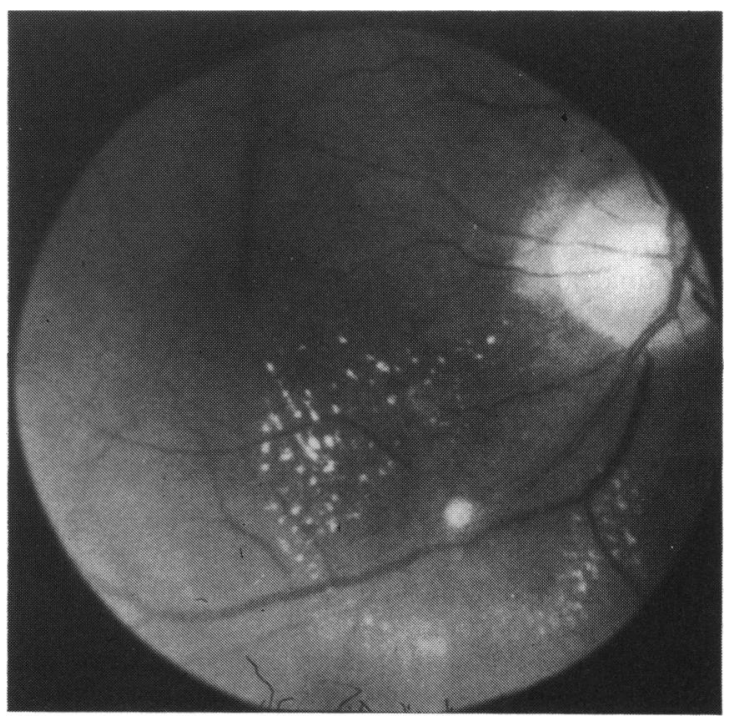

Fig. 2 Fundus photograph of an exudative macroaneurysm. Hyalinised, pale macroaneurysm is located at arterial bifurcation and surrounded by retinal oedema. Exudate is approaching the fovea. 


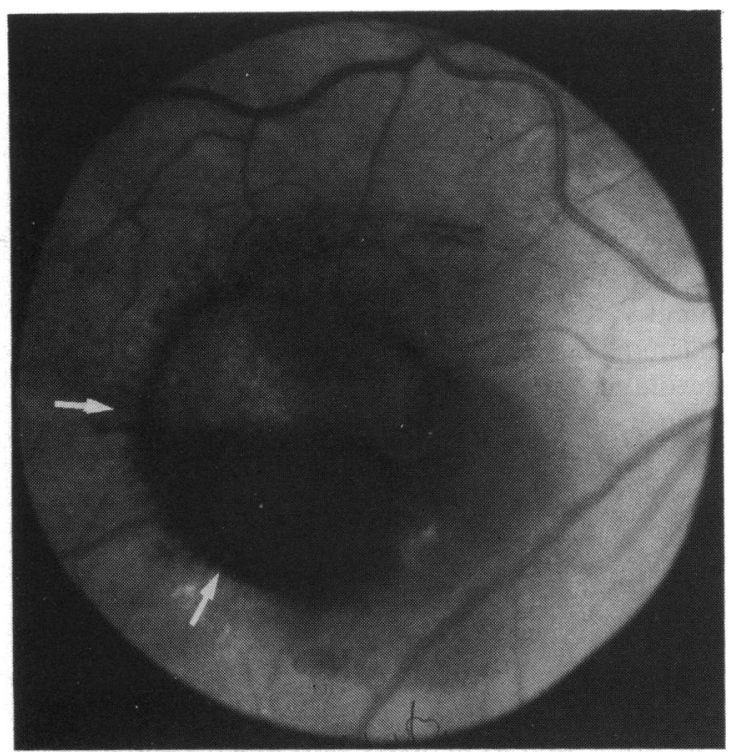

Fig. 3A

Fig. 3 Fundus photograph (A) shows haemorrhagic neuroretinal detachment (arrows) with adjacent subretinal haemorrhage. Venous phase of fluorescein angiogram (B) shows macroaneurysm partially masked by overlying haemorrhage, with proximal arterial constriction.

the MA sufficient to obtain mild to moderate retinal blanching. Burns were then placed on the aneurysm itself using similar intensity until the MA was blanched. Treatments employed a spot size of 100 to $200 \mu \mathrm{m}$ with a duration of 0.1 to 0.2 seconds; nine MAs required an average of 52 burns, while two

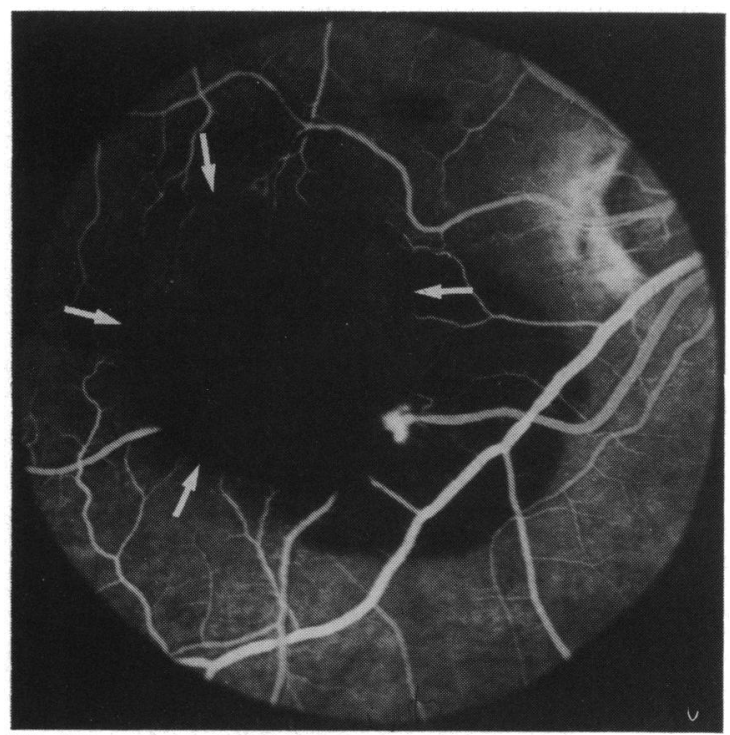

Fig. 3B others needed over 300 burns each. The intensity and number of burns required depend on media clarity and local tissue swelling and uptake characteristics.

\section{Results}

A total of 44 MAs were identified in 43 eyes of 40 patients, and in 37 patients the MAs were single. Three patients had more than one MA, with bilateral involvement in all $3(7 \cdot 5 \%)$.

Sex. A definite female excess was noted, with 28 females $(70 \%)$ and 12 males. The age specific incidence of MAs in the general population was greater for females (Fig. 4).

Age. The age at presentation ranged from 55 to 89 years, with a mean of $66 \cdot 1$ years. The incidence of MAs was found to rise with age (Fig. 4).

Clinical appearance and examination of stereo colour photographs disclosed a set of clinical signs in $86 \%$ of affected eyes which included a local retinal arterial dilatation surrounded by a ring of hyaline change and intraretinal haemorrhage with overlying retinal oedema. In some patients these signs were partly masked by overlying retinal haemorrhage, but in most an accurate diagnosis was possible from examination of the stereo colour photographs alone. Serous neuroretinal detachment was seen in four patients, with macular involvement in two. In most patients the classification as haemorragic or exudative was obvious. In seven patients with haemorrhagic MAs a minor degree of exudate was seen about the peripheral margins of retinal haemorrhage, and two of these later required laser treatment for macular oedema or exudate. Subretinal haemorrhage was present in 20 of the 21 eyes with haemorrhagic MAs. This gradually resorbed over a period of months, leaving a variable degree of pigment

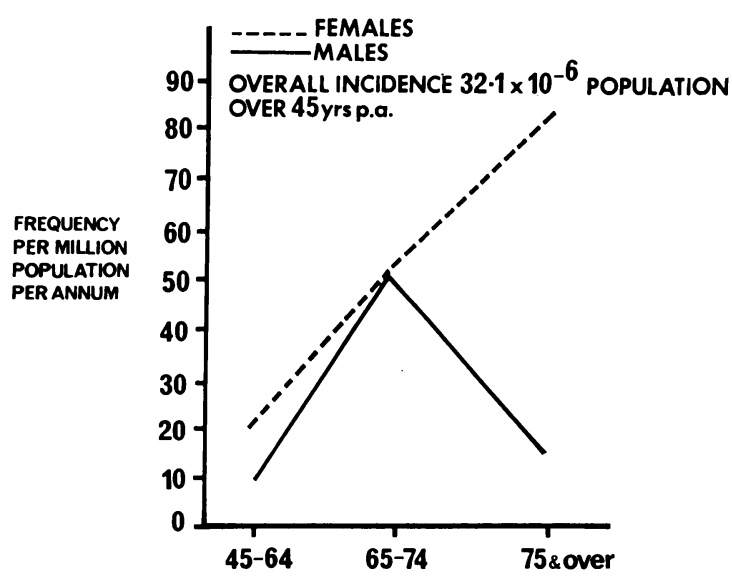

Fig. 4 Age specific incidence of macroaneurysms as calculated from catchment population. 


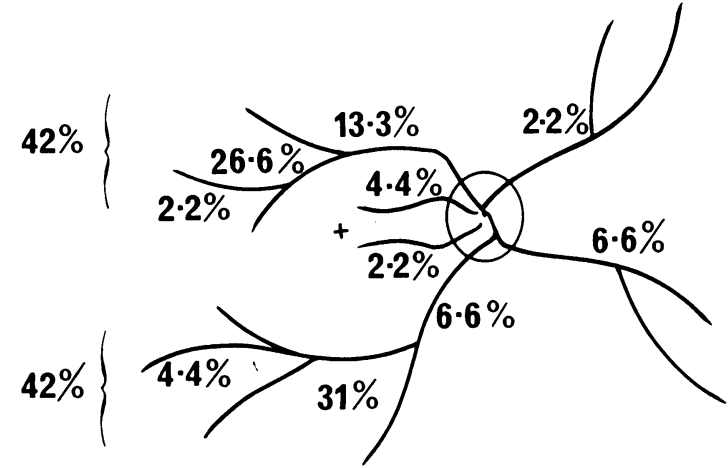

Fig. 5 Distribution of retinal arterial macroaneurysms.

epithelial disturbance and subretinal scarring in its wake. Haemorrhagic MAs resolved spontaneously in all but two patients, both of whom suffered recurrent bleeds. One of these had received laser photocoagulation to the MA some weeks before recurrent haemorrhage.

Site. The right eye was affected in 17 patients and the left in 20. The remaining three patients had bilateral involvement. MAs were as frequent on the superotemporal vessels as on the inferotemporal vessels (Fig. 5). Most.MAs (84\%) were seen on the temporal vascular arcades. Haemorrhagic MAs were located significantly closer to the optic disc $(40 \%$ within 1.5 disc diameters) as compared with other MAs $(16 \%, p<0.05)$. MAs were commonly found in association with arterial bifurcations or arteriovenous crossings.

Presentation. The commonest presenting feature was acute loss of vision, either central or generalised due to haemorrhage, and occurred in 21 eyes of 20 patients $(52 \cdot 5 \%)$. Visual loss was central as a result of macular haemorrhage in 15 eyes, and was generalised owing to vitreous haemorrhage in six. The mean duration of symptoms before presentation in haemorrhagic MAs was 1.4 weeks.

A total of 13 eyes of 13 patients $(32.5 \%)$ were found to have exudative MAs. These patients had a significantly longer duration of symptoms before presentation (mean 16.6 weeks, $\mathrm{p}<0.05$ ), with gradual and often progressive central visual impairment. No patient in this group experienced late haemorrhage or recurrent exudation after apparent resolution of the MA. Four patients had a minor degree of associated intraretinal haemorrhage. A further patient had a significant degree of subretinal haemorrhage, though macular exudate was the major feature.

A total of 11 quiescent MAs were identified in 10 eyes of eight patients. One eye included two quiescent MAs. One patient had a quiescent MA which subsequently ruptured, and this patient is included in the haemorrhagic group for analysis. No other quiescent MAs developed exudation or haemorrhage. Quiescent MAs were discovered incidentally in three patients who presented with retinal vein occlusions, two with macular degeneration, one with diabetic retinopathy, a fellow eye to a haemorrhagic MA, and one patient with lens opacities.

Visual outcome. Initial and final visual acuities are summarised in Figs. 6 and 7. Initial visual acuities of counting fingers or worse were common in haemorrhagic MAs $(52 \%)$ but unusual in exudative MAs $(15 \%)$. In general the outcome was relatively benign, with $54 \%$ of exudative MAs and $48 \%$ of haemorrhagic MAs retaining an acuity of $6 / 12$ or better. Poor visual outcome (counting fingers or worse) was seen in $24 \%$ of haemorrhagic MAs and $15 \%$ of exudative MAs and was related to the severity and duration of macular involvement. Two eyes with exudative MAs
Fig. 6 Initial and final visual acuities in eyes with quiescent (dashed lines) and exudative (continuous lines) macroaneurysms: 22 eyes.

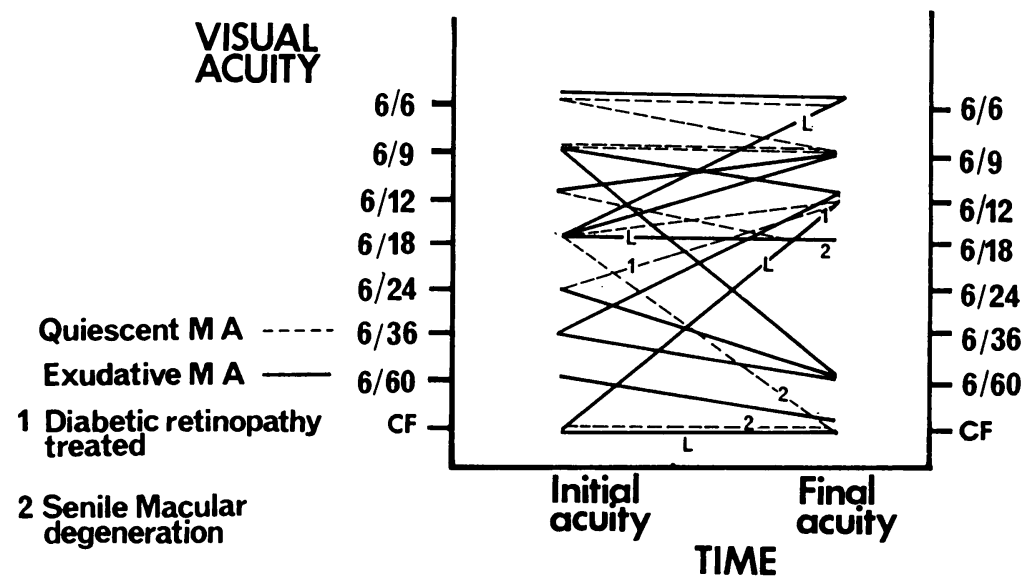




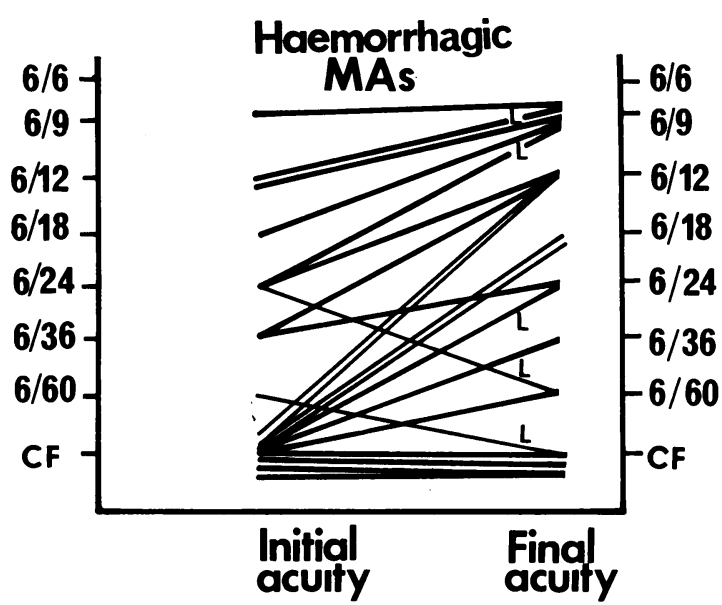

Fig. 7 Initial and final visual acuities in eyes with haemorrhagic macroaneurysms: 21 eyes. $\mathrm{L}=$ laser treated eye.

suffered a reduction in visual acuity of two or more lines owing to macular exudation; in both cases laser treatment had been recommended but was refused. Only two eyes with quiescent MAs suffered reduced acuity, due to senile macular degeneration in both (Fig. 6). The causes of poor final visual acuity are summarised in Table 1. When laser treated groups were compared with untreated groups for different types of MA, no difference in visual outcome was found.

Duration. Follow-up visits for all MAs ranged from a minimum of six months to seven and a half years, with a mean of two years and five months. The duration of MA patency from diagnosis to resolution could be established in seven treated and 14 untreated cases. The total duration of MA patency was significantly shorter in laser treated cases (mean 3.5 months) as compared with untreated cases (mean 13.6 months, $p=0 \cdot 006$, Fisher's exact test), MAs in six out of seven treated eyes resolved within one month of laser treatment, as proved on posttreatment angiography. The duration of untreated MAs ranged from three months to seven years. Haemorrhagic MAs resolved spontaneously in all but two patients, both of whom suffered recurrent haemorrhage $(5 \%)$. One of this pair had received laser photocoagulation to the MA.

Fluorescein findings. Angiography showed that MA sizes were variable, with larger MAs on the

Table 1 Causes of poor final visual acuity (counting fingers orless)

Macular haemorrhage with pigment epithelial scars

Macular exudate

Persistent vit reous hacmorrhage

Senile macular degeneration
4 cyes

2 cyes

1 cyc

2 cyes

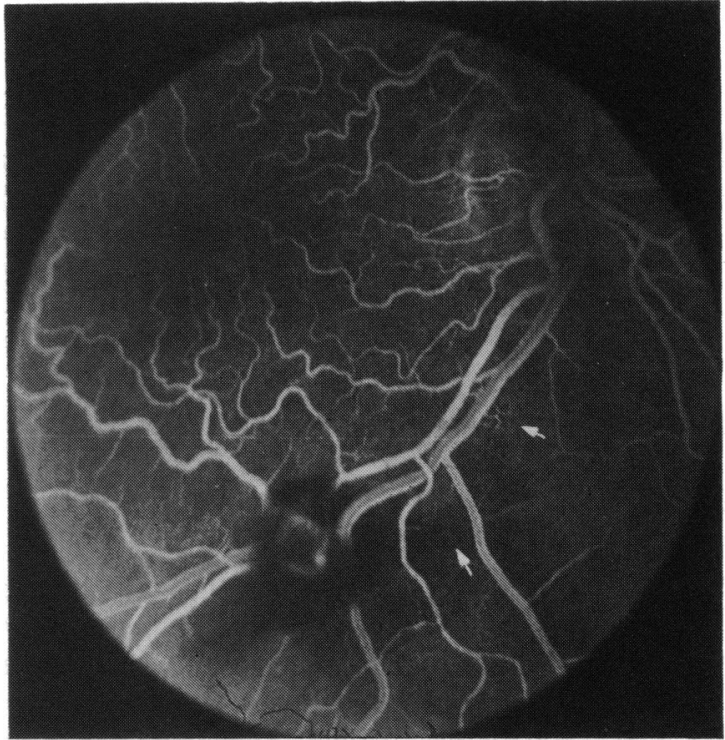

Fig. 8 Fluorescein angiogram in a patient with vitreous haemorrhage from a macroaneurysm discloses multiple areas of capillary telangiectasia and coarsening of the capillary network (arrows)

larger arteries. Aneurysms appeared to be fusiform or saccular. Fusiform MAs showed rapid filling in the early arterial phase and involved most of the arterial circumference. Saccular MAs showed minimal early filling, with good filling in the middle or late phases of the angiogram. The rate of filling seemed to be determined by the size of the aneurysm neck. Fluorescence of MAs was not necessarily homogeneous and many showed irregular inhomogeneous filling, thought to represent intraluminal clot formation. Occasionally the MA was masked by overlying blood, but subsequent angiograms demonstrated the MA. Focal areas of capillary dilatation, telangiectasia, or closure were seen in $48 \%$ of eyes. Although fine capillary changes were frequently seen in the retina adjacent to the $\mathrm{MA}$, in many patients these capillary abnormalities were also located well away from the MA (Fig. 8).

Previous fundus photographs were available from 10 patients, six of whom had had previous angiography for a variety of conditions, most frequently retinal vein occlusion. Careful examination of these angiograms failed to disclose any reliable indicator of future aneurysm formation.

Associated ocular disease. Retinal vein occlusions were seen in the same or fellow eye of 13 patients $(32.5 \%)$, one of whom had a central retinal vein occlusion. A further patient had a branch artery occlusion. Other associated ocular diseases included drusen (19 patients), lens opacities (nine), and epiretinal membranes (two). Retinal arterial plaques 
were seen in the same or fellow eye of eight patients $(20 \%)$, while irregular arterial calibres were noted in 16 patients $(40 \%)$. Patients with haemorrhagic MAs had significantly fewer associated retinal vein occlusion $(15 \%)$ as compared to non-haemorrhagic MAs $(50 \%, \mathrm{p}<0 \cdot 05)$. Half the vein occlusions were in the same eye as affected by the MA. In three branch vein occlusions the MA was situated at the point of vein occlusion, suggesting a causal role in the genesis of the occlusion. Two other MAs were found in the area of retina involved by vein occlusions.

Systemic disease (Fig. 4). Of the 18 patients examined in the medical clinic 17 were found to have evidence of systemic vascular or relevant metabolic disease. A survey of the patients general practitioners found that, of the remaining 22 patients, only four had no known vascular disease. Systolic blood pressures of $200 \mathrm{mmHg}$ or more were more frequent in patients with haemorrhagic MAs $(40 \%)$ than with other MAs (20\%), but the difference was not significant. Blood packed cell volume (haematocrit) levels were available in 20 of our patients and were compared with those in a group of 40 cataract patients matched for age and sex. Haematocrits over $45 \%$ were significantly more frequent in MA patients $(55 \%)$ than controls $(20 \%, \mathrm{p}<0 \cdot 05)$. A history of smoking was available in 15; four were non-smokers and two others had not smoked within the past five years. Two patients died during the study period, both of complications of vascular disease, yielding a mortality of $4 \cdot 8 \%$. One of these patients died before six months of follow-up and is therefore not included for further analysis.

\section{Discussion}

We found that MAs are still often misdiagnosed despite a number of reports describing their clinical features. ${ }^{1.21232+27}$ We emphasise that in most MAs the dominant feature is haemorrhage into the retinal or vitreous spaces and, less commonly, retinal oedema or exudate. These changes may be widespread and often partly obscure the underlying MA, which is therefore easily overlooked. Thus haemorrhagic MAs often masquerade as disciform macular lesions, and MAs with exudation may be misdiagnosed as branch vein occlusions or diabetic retinopathy. Further diagnostic difficulties may arise because branch vein occlusion, senile macular degeneration, or diabetic retinopathy may be seen in these patients and the presentation ascribed to these conditions. It is most important to differentiate these entities because of the very different treatments available. The diagnosis of MA should be considered in all elderly patients with intraocular haemorrhage, retinal oedema, or exudate, particularly if atypical features are present. Subretinal haemorrhage in MAs is typically extensive, dense, overlies an artery, is not centred on the macula, and has vitreous extension. Careful fundus examination supplemented by fluorescein angiography, which may have to be repeated several times, usually establishes the diagnosis.

The age range and sex ratios seen in the study are almost identical to those found on pooling those recorded in the world literature. ${ }^{1-27}$ The predominance of females in this study is not due only to the excess of females in the older population (see Fig. 1). Cerebral aneurysms are most frequent in women after the age of 40, and it is likely that similar pathogenetic factors operate in these two types of aneurysm.

Palestine and colleagues' classification ${ }^{23}$ of MAs did not differentiate between different types of macular involvement. Our classification was simple and identified groups of patients who differed in terms of visual outcome and associated diseases. Although several MAs had mixed haemorrhagic and exudative features, it was usually not difficult to determine the major feature. Subretinal macular haemorrhage is not remediable by laser treatment and usually results in severe visual loss. Macular oedema or exudate on the other hand is eminently treatable, particularly if seen early. Clearly quiescent MAs had the best outcome.

Isolated MAs are almost always seen in the elderly. Aging is associated with replacement of the smooth muscle of the arterial media by collagen and an increase in intimal collagen. The arteries lose their elastic recoil and become rigid and relatively dilated. These changes are similar to those seen in the sclerotic phase of hypertensive retinopathy, and Garner and Klintworth ${ }^{2 x}$ noted that many age related changes may be the result of haemodynamic stress.

Haemorrhagic MAs were located significantly closer to the optic disc than other MAs. Arteries close to the disc have larger diameters and increased flow rates than peripheral vessels. These factors will increase transmural stress in these arteries, and may contribute to haemorrhage.

MAs frequently occur at arteriovenous crossings. At the point where arterial and venous walls are in contact the adventitial layer is absent, and the two vessels share a common coat. ${ }^{29}$ The arterial wall has less structural support at this point and may be prone to aneurysm formation. Any associated venous disease may cause local arterial wall damage, contributing to MA formation.

Retinal vein occlusions are frequently seen in association with MAs, and are also described in other arterial diseases such as Coats' disease ${ }^{\text {it }}$ and Leber's miliary aneurysms." They were uncommon in 
association with haemorrhagic MAs but commonly seen in association with non-haemorrhagic MAs. This suggests that different factors are important in MA rupture, and mechanical factors may be significant. Perhaps these associations reflect the differing effects of widespread retinal vascular disease on high pressure vessels (arterial aneurysm formation) as compared with low pressure vessels (resulting in vein occlusion).

Hypertension is a prominent feature in MA patients." Hypertensive retinopathy is associated with poor retinal vascular autoregulation, plasma transudation, and subsequent vessel hyalinisation. A rigid, dilated arteriole with abnormal wall results. ${ }^{32} 33$ The higher systolic blood pressures seen in patients with haemorrhagic MAs may have contributed to haemorrhage both by the increased transmural tension and by a greater amplitude of pulsations, causing mechanical vascular damage. We could not determine the patients' activities at the time of rupture in a large enough sample. However, studies of cerebral aneurysms have shown that up to $50 \%$ ruptured during events associated with transient arterial hypertension, emphasising the importance of mechanical events, ${ }^{3+35}$ and studies of experimental aneurysms have emphasised the roles of both hypertension and increased haemodynamic stress. ${ }^{36}$ In this respect macroaneurysms have been compared to Charcot's cerebral aneurysms, which may be implicated in the genesis of cerebrovascular accidents. ${ }^{37}$

A compromised vessel wall of any aetiology may be less able to withstand further insults such as embolic damage, particularly in the presence of a high intraluminal pressure. Although MA formation is often ascribed to the mechanical effects of hypertension, ${ }^{1114}$ several authors have drawn attention to the role of local vascular damage. ${ }^{1318-211}$ Retinal arterial emboli are frequently seen in eyes with MAs, which have been shown to develop at sites of previous embolic occlusion. ${ }^{20}$ Indeed, a detailed study of retinal emboli reported one patient with an arterial exudative lesion 'of unknown significance', and the published photograph was very suggestive of an MA. ${ }^{38}$ We note that the distribution of MAs parallels that described for retinal emboli, ${ }^{38}$ showing a marked preference for the temporal retinal vessels and posterior pole. Histologically, MAs are found in areas of arterial disease, ${ }^{19}$ and one eye had evidence of focal small vascular occlusions. ${ }^{19}$ The frequent finding in our study of focal capillary abnormalities in areas adjacent to and away from MAs may be a sequel of small platelet-fibrin emboli. These emboli can travel far down the arterial tree, breaking into small fragments which occlude vessels and subsequently resorb, leaving focal zones of capillary closure. Vascular remodelling can occur after embolic occlusions, ${ }^{39}$ and this may explain the frequent finding of focal capillary dilatation. These capillaries may leak and cause visual loss if located in the perifoveal area, ${ }^{10}$ though this was not seen in our series.

Systemic vascular disease other than hypertension was a prominent feature of our MA patients as it was in other studies. In some patients the MA was the first manifestation of underlying systemic vascular disease. In a few patients systemic vascular disease was not found at their initial visit, but appeared at later follow-up. We are not aware of haematocrit measurements in previous studies of MAs. Although raised haematocrits have been described in patients with retinal vein occlusions, ${ }^{\text {,1 }}$ this did not account for the raised haematocrits seen in our MA patients. Although our sample was small, the raised haematocrits did not appear to be due to smoking. Raised haematocrits are commonly seen in systemic vascular disease. ${ }^{112}$ The raised haematocrits in our MA patients may reflect their underlying systemic vascular disease.

Although some authors have suggested that MA patients have a high death rate after diagnosis, ${ }^{113}$ this was not our experience. The $4.8 \%$ mortality observed over the study period is not alarming given the ages of our patients.

The management of MA patients requires a full investigation for systemic disease, with particular attention to hypertension and systemic vascular and embolic disease. MAs were seen almost exclusively in the sclerotic phase of hypertension, with evidence of long-standing retinal vascular changes. In the face of long-term retinal vascular damage control of hypertension is not likely to cause resolution of the MA, though it might reduce the risk of haemorrhage.

Ocular management depends on MA behaviour. Haemorrhagic MAs with intact maculae should be observed closely in the initial period, as occasionally patients develop macular exudate requiring treatment. In general these MAs resolve spontaneously and do not require treatment. It has been suggested that haemorrhagic MAs should be treated to prevent recurrent haemorrhage. ${ }^{27}$ Both this and a previous study ${ }^{23}$ have found that recurrent haemorrhage is extremely uncommon, and that there are no reliable indicators of impending haemorrhage. In particular, MA pulsation is not a reliable warning of impending haemorrhage. ${ }^{23}$ On the basis of this evidence we believe that the major indication for treatment of haemorrhagic MAs is the development of macular odema or exudate.

Our study shows that treatment results in a significantly shorter duration of MA patency as compared to untreated cases. Since visual loss from macular oedema or exudate depends on severity and dura- 
tion, early photocoagulation of selected cases should be beneficial. When treated cases were compared with untreated cases, Palestine et al. ${ }^{.3}$ found no improvement in visual outcome after photocoagulation. Case selection is particularly important, as patients with long-standing exudate or oedema are unlikely to improve. This was certainly a factor in our patients and explains our finding that photocoagulation did not improve visual outcome. Complications of photocoagulation included occlusion of the distal arteriole and an increase in exudate accumulation soon after photocoagulation, necessitating great care in the treatment of lesions close to the macula.

Direct photocoagulation of the MA may contribute to occlusion of the distal arteriole and was seen in $27 \%$ of our 11 treated cases. However this event may occur spontaneously in the natural history of an MA, and indeed was found in $25 \%$ of Cleary's untreated cases. ${ }^{11}$ Only a small number of our untreated cases underwent fluorescein angiography after resolution of their MA, and these cannot usefully be compared to our treated group. We found distal arteriolar narrowing in $19 \%$ of our untreated MAs on fluorescein angiography. Most MAs resolve spontaneously, and the process of vascular healing is inevitably accompanied by local vessel wall constriction, seen clearly on angiography. Distal occlusion may occur as a result, and presumably depends on the severity and extent of the repair response.

There are no histological studies of the mechanism of laser induced resolution of a MA. Clinical experience suggests that the argon blue-green laser results in thermal uptake both in the retinal pigment epithelium surrounding the MA (causing injury to the outer vessel wall) and in the haemoglobin and oxyhaemoglobin $^{13}$ in the arterial lumen (causing endothelial injury). The aim of perilesional retinal photocoagulation is to aid resolution of oedema, though there is evidence that photocoagulated retinal pigment epithelium may trigger proliferation of nearby retinal vascular endothelial cells. ${ }^{+1}$ This mechanism could also contribute to resolution of the MA. The immediate vasospasm and reduced flow are superseded by a vascular repair response which results in local vessel wall constriction and MA resolution.

We are grateful to Ms Sue Ford for photographic assistance, to Ms Ruth Tobias for statistical assistance, and to Ms Karen Johnstone for the figures. We thank the consultant ophthalmologists at the Western Ophthalmic Hospital for allowing us to review their cases.

\section{References}

1 Robertson DM. Macroaneurysms of the retinal arteries. Ophthalmology 1973; 77: 55-67.

2 Schulman J, Jampol LM, Goldberg MF. Large capillary aneurysms secondary to retinal venous obstruction. $\mathrm{Br} J$ Ophthalmol 1981; 65: 36-41.
3 Henkind P. Walsh JB. Retinal vascular anomalies: pathogenesis, appearance and history. Trans Ophthalmol Soc UK 1980; 100: 425-33.

4 Doyne RW. Case of peculiar condition of the retina due possibly to the formation of small ancurysms and large extravasation of blood which has become decolourised. Trans Ophthalmol Soc $U K$ 1896; 16: 94

5 Loring FB. Peculiar anatomical development of one of the central arteries of the retina. Trans Am Ophthalmol Soc 1881; 3: $40-2$.

6 Story JB, Benson AH. Aneurysms on retinal vessels in a peculiar case of retinitis. Trans Ophthalmol Soc UK 1883; 3: 108-10.

7 Jennings JE. Aneurysms of the retinal arteries. Am J Ophthalmol 1918; 1: 12-3.

8 Hudomel J. Imre G. Photocoagulation treatment of solitary aneurysm near the macula lutea. Acta Ophthalmol (Kbh) 1973 51: 633-8.

9 Schultz WT, Swan KT. Pulsatile aneurysms of the retinal arterial tree. Am J Ophthalmol 1974; 77: 304-9.

10 Cleary PE. Kohner EM, Hamilton AM, Bird AC. Retinal macroaneurysms. Br J Ophthalmol 1975; 59: 335-61.

11 Cleary PE. Retino-vascular malformations. Trans Ophthalmol Soc UK 1976; 96: 213-5.

12 Gold DH, Walsh JB. Fluorescein angiographic patterns of retinal arterial aneurysms. Proc Int Symp Fluorescein Angiography, Ghent. Doc Ophthalmol Proc Ser 1976; 9: $541-7$.

13 Lewis RA. Norton EWD, Gass JDM. Acquired arterial macroaneurysms of the retina. BrJ Ophthalmol 1976; 60:21-30.

14 Nadel AJ, Gupta KK. Macroaneurysms of the retinal arteries. Arch Ophthalmol 1976; 94: 1092-6.

15 Godel V, Blumenthal M. Regenbogen I. Arterial macroaneurysm of the retina. Ophthalmologica 1977; 175: 125-9.

16 Tobari I, Asso S. Yokoro M. Clinical findings and photocoagulation in eight cases of retinal arterial aneurysms. Jpn J Clin Ophthalmol 1977; 31: 803-9.

17 Yoshiodo H, Sugita T, Yamaguchi Y. Seven cases of retinal macroaneurysms. Jpn J Clin Ophthalmol 1977; 31: 175-86.

18 Asdourian GK, Goldberg MF, Jampol LM, Rabb M. Retinal macroaneurysms. Arch Ophthalmol 1977; 95: 624-8.

19 Fichte C. Streeten BW. Freedman AH. A histopathologic study of retinal arterial aneurysms. Am J Ophthalmol 1978; 85: 509-18.

$20 \mathrm{Khalil}$ M, Lorenzetti DWC. Acquired retinal macroaneurysms. Can J Ophthalmol 1979; 14: 163-8.

21 François J. Acquired macroaneurysms of the retinal arteries. Int Ophthalmol 1979; 1: 153-61.

22 Kayazawa F. Bilateral retinal arterial macroaneurysms. Ann Ophthalmol 1980; 12: 18-22.

23 Palestine AG, Robertson DM, Goldstein BG. Macroaneurysms of the retinal arteries. Am J Ophthalmol 1982; 93: 164-71.

24 Spalter HF. Retinal macroaneurysms: a new masquerade syndrome. Trans Am Ophthalmol Soc 1982; 80: 113-30.

25 Van Nouhuys E, Deutman AF. Argon laser treatment of retinal macroaneurysms. Int Ophthalmol 1980; 2: 45-53.

26 Gold DH, LaPiana FG, Zimmerman LE. Isolated retinal arterial aneurysms. Am J Ophthalmol 1976; 82: 848-57.

27 Abdel-Khalek MN, Richardson J. Retinal macroaneurysm: natural history and guidelines for treatment. Br J Ophthalmol 1986; 70: 2-11.

28 Garner A, Klintworth G. Pathobiology of ocular disease. New York: Dekker, 1982: 1480-525.

29 Wise GN, Dollery CT, Henkind P. The retinal circulation. Hagerstown: Harper and Row, 1971: 28-32.

30 Egerer I, Tasman W, Tomer TL. Coats' disease. Arch Ophthalmol 1974; 92: 109-12

31 Wegener JK. Leber's retinal degeneration with miliary ancurysms. Acta Ophthalmol (Kbh) 1969; 47: 108-14.

32 Jampol LM, White S. Cunha Vaz J. Vitreous fluorophotometry in patients with hypertension. Arch Ophthalmol 1983; 101: $888-90$. 
33 Tso MOM, Jampol LM. Pathophysiology of hypertensive retinopathy. Ophthalmology 1982; 89: 1132-45.

34 Locksley HB. Natural history of subarachnoid haemorrhage, intracranial aneurysm and arteriovenous malformations: based on 6368 cases in the cooperatives study. In: Sahs AL. Perrett GE, Locksley HB, Nishioka $\mathrm{H}$, eds. Intracranial aneurysms and subarachnoid haemorrhage: a cooperative study. Philadelphia: Lippincott, 1969: 37-108.

35 Komatsu S, Scki H, Uneoka K, Takaku A, Suzuki J. Rupturing factors of intracranial aneurysm: season, weather and psychosomatic strain. In: Suzuki J, ed. Cerebral aneurysms: experience in 1000 directly operated cases. Tokyo: Neuron, 1979: 25-31.

36 Sekhar LN, Heros RC. Origin, growth and rupture of saccular aneurysms: a review. Neurosurgery $1981 ; 8$ : 248-60.

37 Russell RWR. Observations on intracranial aneurysms. Brain 1963; 86: 425-42.
38 Arruga J, Saunders MD. Ophthalmic findings in 70 patients with evidence of retinal embolism. Ophthalmology 1982;89: 1336-47.

39 Klein R, Klein B, Henkind P, Bellhorn R. Retinal collateral vessel formation. Invest Ophthalmol Vis Sci 1971; 10: 471.

40 Trope GE, Lowe GDO, McArdle BM, et al. Abnormal blood viscosity and haemostasis in long-standing retinal vein occlusion. Br J Ophthalmol 1983; 67: 137-42.

41 Lowe GDO, Forbes CD. Blood rheology and thrombosis. Clin Haematol 1981; 10: 343-67.

42 Lowe GDO. Laboratory evaluation of hypercoagulability. Clin Haematol 1981; 10: 407-42.

43 L'Esperance FA Jr. Ophthalmic lasers: photocoagulation, photoradiation and surgery. St Louis: Mosby, 1983: 93.

44 Clover MMG. Laser treatment of diabetic maculopathy and the implications for retinal vascular barriers. PhD thesis: University of London, 1984.

Accepted for publication 26 September 1986. 